\title{
Local transformation of mixed states of two qubits to Bell diagonal states
}

\author{
Li-Xiang Cen ${ }^{1 *}$, Nan-Jian $\mathrm{Wu}^{1}$, Fu-Hua Yang ${ }^{1}$, and Jun-Hong $\mathrm{An}^{2}$ \\ ${ }^{1}$ NLSM, Institute of Semiconductors, Chinese Academy of Sciences, Beijing 100083, P.R. China, \\ ${ }^{2}$ Department of Modern Physics, Lanzhou University, Lanzhou 730000, P.R.China
}

The optimal entanglement manipulation for a single copy of mixed states of two qubits is to transform it to a Bell diagonal state. In this paper we derive an explicit form of the local operation that can realize such a transformation. The result obtained is universal for arbitrary entangled two-qubit states and it discloses that the corresponding local filter is not unique for density matrices with rank $n=2$ and can be exclusively determined for that with $n=3$ and 4 . As illustrations, a four-parameters family of mixed states are explored, the local filter as well as the transformation probability are given explicitly, which verify the validity of the general result.

Entanglement manipulation is an important issue in the studies of quantum information theory. On the one hand, it is related to the basic problem of which tasks one can accomplish with a given resource of entanglement [1][7]. On the other hand, since most applications of quantum information theory require the maximally entangled state for faithful transmission of quantum data, it is necessary to develop the special technique of entanglement manipulation which uses local quantum operations and classical communication (LOCC) to produce states with the amount of entanglement as great as possible from partly entangled states [2,3].

There has been much attention recently concerning the entanglement manipulation of a single copy of mixed twoqubit states [8]- [14. A basic measure of entanglement of mixed states is the entanglement of formation, which is intended to quantify the entanglement resources needed to create the states [15]. Given a two-qubit density matrix $\rho$, the entanglement of formation is defined as

$$
E(\rho)=\min \sum_{i} p_{i} E\left(\psi_{i}\right),
$$

where the minimum is taken over all decompositions of $\rho$ into pure states

$$
\rho=\sum_{i} p_{i}\left|\psi_{i}\right\rangle\left\langle\psi_{i}\right|
$$

and $E\left(\psi_{i}\right)$ is the entropy of entanglement of the pure state $\left|\psi_{i}\right\rangle$. Although the expected entanglement cannot be increased by any LOCC actions, one can gamble, with risk of failure for some probabilities, on obtaining states with more entanglement from less entangled states [2]. It was proven by Kent etc. [11 that under LOCC operations, the entanglement of formation of a single copy of

*Electronic address: cenlx@red.semi.ac.cn two-qubit states exists an upper bound, the maximum extractable entanglement, and the corresponding state is a Bell diagonal state which is unique up to local unitary transformations. In this stage, a critical problem is to calculate the detailed LOCC operations and determine the probability of success of such a transformation for given two-qubit states.

The local operation on the two-qubit state can be studied in a real matrix parameterization representation of the state, i.e., the Horodecki representation [16]. In a recent paper 13], it was shown that local operators performed on two qubits correspond to left and right multiplication by a Lorentz matrix, respectively, and useful results have been derived from such a point of view. However, until now, the above problem is still open and the distinct form of the LOCC actions that realize the optimal transformation is lack. In the presented paper we investigate this problem in a particular representation, the "Wootters representation" of two-qubit states [17]. We shall show, by introducing an associated operatora squared quantity of the local filter, the explicit form of the optimal transformation as well as the probability can be derived for arbitrary entangled two-qubit states. Moreover, the result displays that the corresponding local filter is not unique for density matrices with rank $n=2$ and can be exclusively determined for that with $n=3$ and 4 . As illustrations, a four-parameters family of mixed states shall be explored, the local filter and the transformation probability are given explicitly, so that the validity of the general result is verified.

Main result.-Consider the local operations on a single copy of two-qubit states. Recall that the local operator can be written as a unitary transformation multiplying a Hermitian filtering operator 10]. One can, without loss of generality, describe the transformation as

$$
\rho \rightarrow \rho^{\prime}=\frac{f_{A} \otimes f_{B} \rho f_{A} \otimes f_{B}}{\operatorname{tr}\left(f_{A}^{2} \otimes f_{B}^{2} \rho\right)} .
$$

Here, we have neglected the local unitary transformation since they shall preserve the entanglement. The local filters $f_{A}$ and $f_{B}$ are given by 10,12

$$
\begin{gathered}
f_{A}=\frac{1}{1+a}\left(I_{2}+a \mathbf{m} \cdot \sigma\right), \\
f_{B}=\frac{1}{1+b}\left(I_{2}+b \mathbf{n} \cdot \sigma\right),
\end{gathered}
$$

where $\mathbf{m}, \mathbf{n}$ are unit vectors and the parameters $a$ and $b$ satisfy $0 \leq a \leq 1,0 \leq b \leq 1$. Above expressions have excluded the trivial cases of the filters with the determinants $\operatorname{det}\left(I_{2}-f_{A(B)}^{2}\right)>0$ which differentiate from the 
operators (4) and (5) only by a coefficient less than 1 . Introduce an associated operator of the local filter $f_{A} \otimes f_{B}$

$$
F_{A B} \equiv \frac{f_{A}^{2} \otimes f_{B}^{2}}{\operatorname{det} f_{A} \operatorname{det} f_{B}} .
$$

This quadratic form $F_{A B}$ is equivalent to the local filter $f_{A} \otimes f_{B}$ in a sense that once $F_{A B}$ is provided, the filtration parameters $a, b, \mathbf{m}$, and $\mathbf{n}$ can be uniquely determined from Eqs. (4)-(6). It can be easily checked, from the relation $f_{A(B)} \tilde{f}_{A(B)}=\operatorname{det} f_{A(B)} I_{2}$, that the operator $F_{A B}$ has the property

$$
F_{A B} \tilde{F}_{A B}=I_{2} \otimes I_{2},
$$

where the tilde " " of an operator is defined as $\tilde{F}_{A B} \equiv$ $\sigma_{2} \otimes \sigma_{2} F_{A B}^{*} \sigma_{2} \otimes \sigma_{2}$. In the following, we shall determine the detailed form of $F_{A B}$ for the optimal entanglement manipulation which transforms the two-qubit state $\rho$ into a Bell diagonal state.

We make use of the so-called "Wootters' representation", i.e., the particular decomposition of the two-qubit state 17]

$$
\rho=\sum_{i=1}^{n}\left|x_{i}\right\rangle\left\langle x_{i}\right|
$$

Here, $n \leq 4$ is the rank of $\rho$. The states $\left|x_{i}\right\rangle$ satisfy

$$
\left\langle x_{i} \mid \tilde{x}_{j}\right\rangle=\lambda_{i} \delta_{i j}, \quad\left|\tilde{x}_{i}\right\rangle=\sigma_{2} \otimes \sigma_{2}\left|x_{i}^{*}\right\rangle,
$$

and the $\lambda_{i}$ s are the eigenvalues, in decreasing order, of the Hermitian matrix $R(\rho) \equiv \sqrt{\sqrt{\rho} \tilde{\rho} \sqrt{\rho}}$. A combination of these symbols defines the concurrence for the entangled state $\rho: C(\rho)=\lambda_{1}-\lambda_{2}-\lambda_{3}-\lambda_{4}$. It turns out that the above representation is very useful to determine the operator $F_{A B}$. Namely, we have

Proposition.-An entangled two-qubit state $\rho$ given by (8) with $\lambda_{n}>0$ can be transformed to a Bell diagonal state by the local filter $f_{A} \otimes f_{B}$ if and only if the associated operator $F_{A B}$ satisfies

$$
\left\langle x_{i}\left|F_{A B}\right| x_{i}\right\rangle=\left\langle x_{i} \mid \tilde{x}_{i}\right\rangle, \quad i=1, \cdots, n .
$$

Relation (10) implies that the normalized states

$$
\left|E_{i}\right\rangle \equiv \frac{f_{A} \otimes f_{B}\left|x_{i}\right\rangle}{\sqrt{\left\langle x_{i}\left|f_{A}^{2} \otimes f_{B}^{2}\right| x_{i}\right\rangle}}, \quad i=1, \cdots, n
$$

have the "tilde inner products" $\left\langle E_{i} \mid \tilde{E}_{i}\right\rangle=1$, thus $\left|E_{i}\right\rangle=$ $\left|\tilde{E}_{i}\right\rangle$. This yields explicitly

$$
\left\langle x_{i}\left|F_{A B}\right| x_{j}\right\rangle=\left\langle x_{i} \mid \tilde{x}_{j}\right\rangle=0, \quad i \neq j .
$$

The sufficiency of the proposition can be shown directly. Following the fact that the states $\left|E_{i}\right\rangle(i=$ $1, \cdots, n$ ) have the concurrence 1 , that is, they are a set of maximally entangled states of qubits $\mathrm{A}$ and $\mathrm{B}$, one can conclude that the transformed state $\rho^{\prime}$ has completely random local density matrices $\operatorname{tr}_{A} \rho^{\prime}=\operatorname{tr}_{B} \rho^{\prime}=\frac{1}{2} I_{2}$, thus is Bell diagonal. To prove the converse statement, we use the fact that the Bell diagonal state has the property [12]

$$
\operatorname{tr} R(\rho)=\sum_{i=1}^{n} \lambda_{i}=1
$$

Now suppose the transformed state

$$
\rho^{\prime}=\frac{\sum_{i=1}^{n} f_{A} \otimes f_{B}\left|x_{i}\right\rangle\left\langle x_{i}\right| f_{A} \otimes f_{B}}{\operatorname{tr}\left(\rho f_{A}^{2} \otimes f_{B}^{2}\right)}=\sum_{i=1}^{n} p_{i}\left|E_{i}\right\rangle\left\langle E_{i}\right|
$$

is Bell diagonal. Here, $\left|E_{i}\right\rangle$ is given by Eq. (11) and $p_{i}$ is the probability of it in the decomposition. Direct observation can be found that the states $\left|E_{i}\right\rangle(i=1, \cdots, n)$ have tilde inner products $\left\langle E_{i} \mid \tilde{E}_{j}\right\rangle=\left\langle E_{i} \mid \tilde{E}_{i}\right\rangle \delta_{i j}$ with $\left\langle E_{i} \mid \tilde{E}_{i}\right\rangle$ positive real numbers. Thus we have

$$
\sum_{i=1}^{n} \operatorname{tr} R\left(\rho^{\prime}\right)=\sum_{i=1}^{n} p_{i}\left\langle E_{i} \mid \tilde{E}_{i}\right\rangle=1 .
$$

This yields that $\left\langle E_{i} \mid \tilde{E}_{i}\right\rangle=1$ for all $i=1, \cdots, n$, then the relation (10).

The analysis presented above also indicates that the two-qubit states with $\lambda_{n}=0$ cannot be transformed into Bell diagonal states by any local actions efficiently. Note that $\lambda_{n}=0$ can only be possible when $n=3$ and $n=2$ [18]. In such cases, $\left|x_{n}\right\rangle$ is a factorizable state of qubits $\mathrm{A}$ and $\mathrm{B}$, and cannot be transformed to the maximally entangled state (corresponding to $\left|E_{n}\right\rangle$ in the above description) by any local filtering operations. The best one can do, is to reduce the probability of this term to the infinitesimal. This process, as has been pointed out in Ref. [13], transforms the state asymptotically to a Bell diagonal state with a lower rank and an infinitesimal probability.

Equations (10) and (12) suggest a distinct way to construct the associated local operator $F_{A B}$. Knowing that the rank $n=1$ corresponds to pure states and they can be purified by the Procrustean protocol [2], we discuss in the following the cases of $n=4,3$, and 2, respectively.

1. $n=4$. In this case, the states $\left\{\left|x_{i}\right\rangle, i=1, \cdots, 4\right\}$ compose a complete set of non-orthogonal bases of the 4-dimensional Hilbert space of the two qubits. Equations (10) and (12) thus provide a clear representation of the operator $F_{A B}$ in these bases. Recalling $\left\{\left|\tilde{x}_{i}\right\rangle, i=1, \cdots, 4\right\}$ the set of biorthogonal bases to $\left\{\left|x_{i}\right\rangle, i=1, \cdots, 4\right\}$, we can present explicitly

$$
F_{A B}=\sum_{i=1}^{4} \frac{\left|\tilde{x}_{i}\right\rangle\left\langle\tilde{x}_{i}\right|}{\left\langle x_{i} \mid \tilde{x}_{i}\right\rangle} .
$$

To derive detailed expressions for the local filters $f_{A}$ and $f_{B}$, we need to factorize the operator given above. This shall be shown later in this section. 
2. $n=3$. Apparently, there exists a unique state $\left|x_{4}\right\rangle$ in the 4-dimensional Hilbert space which is orthogonal to the three linearly-independent states $\left\{\left|\tilde{x}_{i}\right\rangle, i=1,2,3\right\}$. Noticing the relation $\left|E_{i}\right\rangle=\left|\tilde{E}_{i}\right\rangle(i=1,2,3)$, one can find that

$$
\left\langle x_{4}\left|F_{A B}\right| x_{i}\right\rangle=\left\langle x_{i}\left|F_{A B}\right| x_{4}\right\rangle=0, \quad i=1,2,3 .
$$

Suppose $\left\langle x_{4}\left|F_{A B}\right| x_{4}\right\rangle=t_{44}$. The operator $F_{A B}$ can be expressed as

$$
F_{A B}=\sum_{i=1}^{3} \frac{\left|\tilde{x}_{i}\right\rangle\left\langle\tilde{x}_{i}\right|}{\left\langle x_{i} \mid \tilde{x}_{i}\right\rangle}+t_{44} \frac{\left|\tilde{x}_{4}\right\rangle\left\langle\tilde{x}_{4}\right|}{\left\langle x_{4} \mid \tilde{x}_{4}\right\rangle^{2}} .
$$

Substituting this expression into Eq. (7), one obtains $t_{44}=\left\langle x_{4} \mid \tilde{x}_{4}\right\rangle$. Thus in this case the local operator $F_{A B}$ is also unique and can be expressed uniformly as (16).

3. $n=2$. Similarly, provided the states $\left\{\left|x_{1}\right\rangle\right.$, $\left.\left|x_{2}\right\rangle\right\}$, one can easily construct the complete set $\left\{\left|x_{i}\right\rangle, i=\right.$ $1, \cdots, 4\}$ in the 4-dimensional Hilbert space of the two qubits. In this case, the states $\left|x_{3}\right\rangle$ and $\left|x_{4}\right\rangle$ can be chosen variously. Suppose $\left\{\left|x_{i}\right\rangle, i=1, \cdots, 4\right\}$ is one of the selected sets satisfying (9). Based on an analysis similar to the previous case, we can present the form of the Hermitian operator $F_{A B}$ as

$$
\begin{aligned}
F_{A B} & =\sum_{i=1}^{2} \frac{\left|\tilde{x}_{i}\right\rangle\left\langle\tilde{x}_{i}\right|}{\left\langle x_{i} \mid \tilde{x}_{i}\right\rangle}+\sum_{i=3}^{4} t_{i i} \frac{\left|\tilde{x}_{i}\right\rangle\left\langle\tilde{x}_{i}\right|}{\left\langle x_{i} \mid \tilde{x}_{i}\right\rangle^{2}} \\
& +t_{34}\left(\frac{\left|\tilde{x}_{3}\right\rangle\left\langle\tilde{x}_{4}\right|}{\left\langle x_{3} \mid \tilde{x}_{3}\right\rangle\left\langle x_{4} \mid \tilde{x}_{4}\right\rangle}+\frac{\left|\tilde{x}_{4}\right\rangle\left\langle\tilde{x}_{3}\right|}{\left\langle x_{3} \mid \tilde{x}_{3}\right\rangle\left\langle x_{4} \mid \tilde{x}_{4}\right\rangle}\right) .
\end{aligned}
$$

Direct calculations from the restriction (7) can show that

$$
t_{34}=0, \quad t_{33}=\left\langle x_{3} \mid \tilde{x}_{3}\right\rangle, \quad t_{44}=\left\langle x_{4} \mid \tilde{x}_{4}\right\rangle .
$$

This yields again the form of $(16)$. The operator $F_{A B}$ now is not unique due to different selections of the states $\left\{\left|x_{3}\right\rangle,\left|x_{4}\right\rangle\right\}$. Specifically, they can be obtained through constructing the states as

$$
\begin{aligned}
\left|x_{3}^{\prime}\right\rangle & =\frac{c_{1}\left|x_{3}\right\rangle}{\sqrt{\left\langle x_{3} \mid \tilde{x}_{3}\right\rangle}}+\frac{d_{1}\left|x_{4}\right\rangle}{\sqrt{\left\langle x_{4} \mid \tilde{x}_{4}\right\rangle}} \\
\left|x_{4}^{\prime}\right\rangle & =\frac{c_{2}\left|x_{3}\right\rangle}{\sqrt{\left\langle x_{3} \mid \tilde{x}_{3}\right\rangle}}+\frac{d_{2}\left|x_{4}\right\rangle}{\sqrt{\left\langle x_{4} \mid \tilde{x}_{4}\right\rangle}}
\end{aligned}
$$

with the complex parameters $c_{i}$ and $d_{i}$ satisfying

$$
\begin{gathered}
c_{1}^{*} c_{2}^{*}+d_{1}^{*} d_{2}^{*}=0, \\
\left(c_{1}^{*}\right)^{2}+\left(d_{1}^{*}\right)^{2}=1, \\
\left(c_{2}^{*}\right)^{2}+\left(d_{2}^{*}\right)^{2}=1 .
\end{gathered}
$$

We now need to show the operator $F_{A B}$ presented in Eq. (16) can always be factorized as a product form of local operators of qubits $\mathrm{A}$ and $\mathrm{B}$. To this end, we subnormalize the states $\left\{\left|\tilde{x}_{i}\right\rangle, i=1, \cdots, 4\right\}$ such that

$$
F_{A B}=\sum_{i=1}^{4}\left|y_{i}\right\rangle\left\langle y_{i}|, \quad| y_{i}\right\rangle=\frac{\left|\tilde{x}_{i}\right\rangle}{\sqrt{\left\langle x_{i} \mid \tilde{x}_{i}\right\rangle}}
$$

The states $\left\{\left|y_{i}\right\rangle\right\}$ have orthogonal normalized tilde inner products

$$
\left\langle y_{i} \mid \tilde{y}_{j}\right\rangle=\delta_{i j}, \quad i, j=1, \cdots, 4 .
$$

Construct a new decomposition of the operator $F_{A B}=$ $\sum_{i=1}^{4}\left|z_{i}\right\rangle\left\langle z_{i}\right|$ with

$$
\begin{aligned}
\left|z_{1}\right\rangle & =\frac{1}{2}\left[\left(\left|y_{1}\right\rangle+\left|y_{2}\right\rangle\right)+i\left(\left|y_{3}\right\rangle+\left|y_{4}\right\rangle\right)\right], \\
\left|z_{2}\right\rangle & =\frac{1}{2}\left[\left(\left|y_{1}\right\rangle+\left|y_{2}\right\rangle\right)-i\left(\left|y_{3}\right\rangle+\left|y_{4}\right\rangle\right)\right], \\
\left|z_{3}\right\rangle & =\frac{1}{2}\left[\left(\left|y_{1}\right\rangle-\left|y_{2}\right\rangle\right)+i\left(\left|y_{3}\right\rangle-\left|y_{4}\right\rangle\right)\right], \\
\left|z_{4}\right\rangle & =\frac{1}{2}\left[\left(\left|y_{1}\right\rangle-\left|y_{2}\right\rangle\right)-i\left(\left|y_{3}\right\rangle-\left|y_{4}\right\rangle\right)\right] .
\end{aligned}
$$

One can check directly that the four states $\left|z_{i}\right\rangle(i=$ $1, \cdots, 4)$ have the concurrence zero, thus are factorizable states of qubits A and B. In detail, there are

$$
\begin{gathered}
\left\langle z_{1} \mid \tilde{z}_{j}\right\rangle=\delta_{2 j}, \quad\left\langle z_{2} \mid \tilde{z}_{j}\right\rangle=\delta_{1 j}, \\
\left\langle z_{3} \mid \tilde{z}_{j}\right\rangle=\delta_{4 j}, \quad\left\langle z_{4} \mid \tilde{z}_{j}\right\rangle=\delta_{3 j} .
\end{gathered}
$$

Now we show that these relations together with the linearly-independent property of the states $\left\{\left|z_{i}\right\rangle, i=\right.$ $1, \cdots, 4\}$ can predict that the operator $F_{A B}$ takes a factorizable form.

Let us first assume that

$$
\left|z_{1}\right\rangle=\alpha_{1}\left|a_{1} b_{1}\right\rangle, \quad\left|z_{2}\right\rangle=\alpha_{2}\left|a_{2} b_{2}\right\rangle
$$

where the local basis $\left|a_{i}\right\rangle$ and $\left|b_{i}\right\rangle$ are normalized. According to relations $\left\langle z_{1} \mid \tilde{z}_{1}\right\rangle=\left\langle z_{3} \mid \tilde{z}_{3}\right\rangle=\left\langle z_{1} \mid \tilde{z}_{3}\right\rangle=0$, one can check that an arbitrary superposition of the two factorizable states $\left|z_{1}\right\rangle$ and $\left|z_{3}\right\rangle$ shall still be a factorizable state of qubits $\mathrm{A}$ and $\mathrm{B}$. So it can be concluded that the state $\left|z_{3}\right\rangle$ must take a form of $\left|z_{3}\right\rangle=\alpha_{3}\left|a_{1} b_{3}\right\rangle$ or $\left|z_{3}\right\rangle=\alpha_{3}\left|a_{3} b_{1}\right\rangle$. Note there are the same relations for the states $\left\{\left|z_{2}\right\rangle,\left|z_{3}\right\rangle\right\}$. Combining these two restrictions gives that the state $\left|z_{3}\right\rangle$ must take the form

$$
\left|z_{3}\right\rangle=\alpha_{3}\left|a_{1} b_{2}\right\rangle
$$

or

$$
\left|z_{3}\right\rangle=\alpha_{3}\left|a_{2} b_{1}\right\rangle
$$

Correspondingly, the similar analysis is applicable to the states $\left\{\left|z_{1}\right\rangle,\left|z_{2}\right\rangle,\left|z_{4}\right\rangle\right\}$. Knowing that the four states $\left|z_{i}\right\rangle$ $(i=1, \cdots, 4)$ are linearly independent, we can obtain the form of the state $\left|z_{4}\right\rangle$ :

$$
\left|z_{4}\right\rangle=\alpha_{4}\left|a_{2} b_{1}\right\rangle
$$

or

$$
\left|z_{4}\right\rangle=\alpha_{4}\left|a_{1} b_{2}\right\rangle
$$

corresponding to the two cases of (28) and (29), respectively. Finally, according to the relation $\left\langle z_{1} \mid \tilde{z}_{2}\right\rangle=$ 
$\left\langle z_{3} \mid \tilde{z}_{4}\right\rangle=1$, the complex coefficients of the states must satisfy $\left|\alpha_{1} \alpha_{2}\right|=\left|\alpha_{3} \alpha_{4}\right|$ for both of these two cases. Now it can be shown directly that the operator $F_{A B}$ takes a factorizable form of local filters of qubits $\mathrm{A}$ and $\mathrm{B}$.

Up to now, we have presented a distinct expression for the associated operator $F_{A B}$ and shown that the local filters $f_{A}$ and $f_{B}$ can be obtained through factorizing $F_{A B}$ straightforwardly. To complete the argument, we need to calculate the probability $P_{f}$ that the LOCC action succeeds. Directly,

$$
P_{f}=\operatorname{tr}\left(\rho f_{A}^{2} \otimes f_{B}^{2}\right)=\operatorname{det} f_{A} \operatorname{det} f_{B} \sum_{i=1}^{n}\left\langle x_{i}\left|F_{A B}\right| x_{i}\right\rangle \text {. }
$$

It can be found, from Eqs. (4)-(6), that the coefficient $\operatorname{det} f_{A}$ det $f_{B}$ is equal to the minimum eigenvalue $\lambda_{\min }^{F}$ of the associated operator $F_{A B}$. Thus we have

$$
P_{f}=\lambda_{\min }^{F} \operatorname{tr} R(\rho) .
$$

Illustration.-We now apply the results derived above to treat the following four-parameters family of mixed states,

$$
\rho=p_{1}\left|\psi_{1}\right\rangle\left\langle\psi_{1}\left|+p_{2}\right| \psi_{2}\right\rangle\left\langle\psi_{2}\left|+p_{3}\right| 00\right\rangle\left\langle 00\left|+p_{4}\right| 11\right\rangle\langle 11| .
$$

Here

$$
\left|\psi_{1}\right\rangle=\alpha|01\rangle-\beta|10\rangle,\left|\psi_{2}\right\rangle=\beta|01\rangle+\alpha|10\rangle
$$

and $\alpha, \beta$ are two positive real numbers satisfying $\alpha^{2}+$ $\beta^{2}=1$. The four states $\left|\psi_{1}\right\rangle,\left|\psi_{2}\right\rangle,|00\rangle$ and $|11\rangle$ compose the orthogonal decomposition of $\rho$ and the corresponding probabilities are assumed to satisfy $p_{1} \geq p_{2}$ and $p_{3} \geq p_{4}$. Exploration of the states (34) should be of universal value: it comprises lower rank states as some of the coefficients $p_{i}$ reduce to zero, so the results acquired should predict the various consequences mentioned above for these reduced cases. We demonstrate these expectations in detail below.

A direct calculation gives the Wootters representation of the state $\rho$ with

$$
\begin{aligned}
& \left|x_{1}\right\rangle=i \cos \theta \sqrt{p_{1}}\left|\psi_{1}\right\rangle-i \sin \theta \sqrt{p_{2}}\left|\psi_{2}\right\rangle, \\
& \left|x_{2}\right\rangle=\sin \theta \sqrt{p_{1}}\left|\psi_{1}\right\rangle+\cos \theta \sqrt{p_{2}}\left|\psi_{2}\right\rangle, \\
& \left|x_{3}\right\rangle=i \frac{\sqrt{2}}{2}\left(\sqrt{p_{3}}|00\rangle+\sqrt{p_{4}}|11\rangle,\right. \\
& \left|x_{4}\right\rangle=\frac{\sqrt{2}}{2}\left(\sqrt{p_{3}}|00\rangle-\sqrt{p_{4}}|11\rangle,\right.
\end{aligned}
$$

and their tilde inner products are

$$
\begin{aligned}
& \lambda_{1}=\left[\alpha^{2} \beta^{2}\left(p_{1}-p_{2}\right)^{2}+p_{1} p_{2}\right]^{1 / 2}+\alpha \beta\left(p_{1}-p_{2}\right), \\
& \lambda_{2}=\left[\alpha^{2} \beta^{2}\left(p_{1}-p_{2}\right)^{2}+p_{1} p_{2}\right]^{1 / 2}-\alpha \beta\left(p_{1}-p_{2}\right), \\
& \lambda_{3}=\lambda_{4}=\sqrt{p_{3} p_{4}} .
\end{aligned}
$$

The parameter $\theta$ in (36) is given by

$$
\theta=\arctan \frac{\sqrt{p_{1} p_{2}}\left(\alpha^{2}-\beta^{2}\right)}{\left[\alpha^{2} \beta^{2}\left(p_{1}-p_{2}\right)^{2}+p_{1} p_{2}\right]^{1 / 2}+\alpha \beta\left(p_{1}+p_{2}\right)} .
$$

Provided the presumption that the considered state $\rho$ has nonzero entanglement of formation (thus nonzero concurrence), the parameters $\alpha, \beta$, and $p_{i}$ s should satisfy

$$
C(\rho)=2\left[\alpha \beta\left(p_{1}-p_{2}\right)-\sqrt{p_{3} p_{4}}\right]>0 .
$$

Now according to the results derived in the preceding section, the local operation of the optimal transformation for the rank- 4 state $\rho$ can be uniquely determined. The factorized form of the associated operator $F_{A B}$ can be obtained by a step-by-step calculation from the proposed approach. The final form of it can be shown as

$F_{A B}=k^{2}\left(\frac{1}{k^{2}} \sqrt{\frac{p_{4}}{p_{3}}}|0\rangle\langle 0|+| 1\rangle\langle 1|\right) \otimes\left(|0\rangle\left\langle 0\left|+\frac{1}{k^{2}} \sqrt{\frac{p_{3}}{p_{4}}}\right| 1\right\rangle\langle 1|\right)$,

where

$$
k \equiv \sqrt{\frac{2}{\lambda_{1}}}\left(\sqrt{p_{1}} \alpha \cos \theta-\sqrt{p_{2}} \beta \sin \theta\right) .
$$

The local filters $f_{A}$ and $f_{B}$ can be easily determined from Eqs. (4)-(6). For example, as the parameters satisfying $\sqrt{p_{4} / p_{3}} \leq \sqrt{p_{3} / p_{4}} \leq k^{2}$, they can be shown as

$f_{A}=\frac{1}{k}\left(\frac{p_{4}}{p_{3}}\right)^{1 / 4}|0\rangle\langle 0|+| 1\rangle\left\langle 1\left|, f_{B}=\right| 0\right\rangle\left\langle 0\left|+\frac{1}{k}\left(\frac{p_{3}}{p_{4}}\right)^{1 / 4}\right| 1\right\rangle\langle 1|$,

and for the case $k^{2} \leq \sqrt{p_{4} / p_{3}} \leq \sqrt{p_{3} / p_{4}}$, they are

$f_{A}=|0\rangle\left\langle 0\left|+k\left(\frac{p_{3}}{p_{4}}\right)^{1 / 4}\right| 1\right\rangle\left\langle 1\left|, f_{B}=k\left(\frac{p_{4}}{p_{3}}\right)^{1 / 4}\right| 0\right\rangle\langle 0|+| 1\rangle\langle 1|$.

The probability of the transformation is given by

$$
P_{f}=\frac{2}{k^{2}}\left\{\left[\alpha^{2} \beta^{2}\left(p_{1}-p_{2}\right)^{2}+p_{1} p_{2}\right]^{1 / 2}+\sqrt{p_{3} p_{4}}\right\}
$$

or

$$
P_{f}=2 k^{2}\left\{\left[\alpha^{2} \beta^{2}\left(p_{1}-p_{2}\right)^{2}+p_{1} p_{2}\right]^{1 / 2}+\sqrt{p_{3} p_{4}}\right\}
$$

corresponding to the above two cases, respectively. The transformed Bell diagonal state can be worked out from Eq. (14). As $p_{2}=0$, it can be shown simply

$\rho^{\prime}=\frac{1}{N}\left[2 p_{1} \alpha \beta\left|\Psi_{-}\right\rangle\left\langle\Psi_{-}\right|+\sqrt{p_{3} p_{4}}\left(\left|\Phi_{+}\right\rangle\left\langle\Phi_{+}|+| \Phi_{-}\right\rangle\left\langle\Phi_{-}\right|\right)\right]$,

where the normalized factor $N=2 p_{1} \alpha \beta+2 \sqrt{p_{3} p_{4}}$ and $\left|\Psi_{-}\right\rangle$and $\left|\Phi_{ \pm}\right\rangle$are Bell states given by 


$$
\begin{aligned}
& \left|\Psi_{-}\right\rangle=\frac{\sqrt{2}}{2}(|01\rangle-|10\rangle), \\
& \left|\Phi_{ \pm}\right\rangle=\frac{\sqrt{2}}{2}(|00\rangle \pm|11\rangle) .
\end{aligned}
$$

The state $\rho^{\prime}$ has the maximal extractable concurrence

$$
C\left(\rho^{\prime}\right)=\frac{p_{1} \alpha \beta-\sqrt{p_{3} p_{4}}}{p_{1} \alpha \beta+\sqrt{p_{3} p_{4}}}=\left[\frac{C(\rho)}{\operatorname{tr} R(\rho)}\right]_{p_{2}=0},
$$

which verifies the formula (12) presented in Ref. 12].

Now let us give a discussion for the degenerate cases.

1. First consider the situation as the parameter $p_{4}$ approaches to zero asymptotically and the limit of it corresponds to a rank 3 density matrix with $\lambda_{3}=0$ (or a rank 2 state with $\lambda_{2}=0$ as $p_{2}=0$ ). In such a case, the relation $\sqrt{p_{4} / p_{3}} \leq k^{2} \leq \sqrt{p_{3} / p_{4}}$ exists and the local filters $f_{A}$ and $f_{B}$ can be obtained from Eq. (40) as

$$
f_{A}=\frac{1}{k}\left(\frac{p_{4}}{p_{3}}\right)^{1 / 4}|0\rangle\langle 0|+| 1\rangle\left\langle 1\left|, f_{B}=k\left(\frac{p_{4}}{p_{3}}\right)^{1 / 4}\right| 0\right\rangle\langle 0|+| 1\rangle\langle 1| .
$$

One can see in the limit of $p_{4} \rightarrow 0, f_{A}$ and $f_{B}$ reduce to projection operators of pure states of the two qubits, respectively, and the probability of the manipulation

$$
P_{f}=2 \sqrt{\frac{p_{4}}{p_{3}}}\left\{\left[\alpha^{2} \beta^{2}\left(p_{1}-p_{2}\right)^{2}+p_{1} p_{2}\right]^{1 / 2}+\sqrt{p_{3} p_{4}}\right\}
$$

becomes zero. This confirms the assertion that any twoqubit density matrix with $\lambda_{n}=0$ cannot be transformed into Bell diagonal state efficiently by invertible local actions.

2. Another notable situation is the rank 2 case with $p_{3}=p_{4}=0$. In this case the expression given by (40) appears to be not valid any more. The analysis proposed previously predicts that there exist various solutions for the local operator, and they can be obtained by establishing the bases $\left\{\left|x_{3}\right\rangle,\left|x_{4}\right\rangle\right\}$ as

$$
\left|x_{3}\right\rangle=i\left(\tau_{1}|00\rangle+\tau_{2}|11\rangle\right),\left|x_{4}\right\rangle=\tau_{1}|00\rangle-\tau_{2}|11\rangle,
$$

where $\tau_{1}$ and $\tau_{2}$ are complex parameters and the multiplication of them satisfies $\tau_{1} \tau_{2}=\left|\tau_{1} \tau_{2}\right|>0$. Similarly, the factorizable form of the associated operator $F_{A B}$ can be worked out

$$
F_{A B}=k^{2}\left(\frac{1}{k^{2}} \frac{\left|\tau_{2}\right|}{\left|\tau_{1}\right|}|0\rangle\langle 0|+| 1\rangle\langle 1|\right) \otimes\left(|0\rangle\left\langle 0\left|+\frac{1}{k^{2}} \frac{\left|\tau_{1}\right|}{\left|\tau_{2}\right|}\right| 1\right\rangle\langle 1|\right) .
$$

In comparison with Eq. (40), the difference is that the ratio $\left|\tau_{2}\right| /\left|\tau_{1}\right|$ in (52) can be an arbitrary positive number, which implies the multiplicity of the local action. As the parameters are chosen that $\left|\tau_{1}\right|=\left|\tau_{2}\right|$, the local action has the optimal probability

$$
P_{f}=2\left[\alpha^{2} \beta^{2}\left(p_{1}-p_{2}\right)^{2}+p_{1} p_{2}\right]^{1 / 2} \times \min \left\{k^{2}, \frac{1}{k^{2}}\right\} .
$$

In conclusion, we have explored the optimal entanglement manipulation for a single copy of two-qubit states. By virtue of a particular representation of the two-qubit state, an explicit form of the LOCC action has been derived to transform the state into Bell diagonal state and the probability of the transformation was also given. The results display that the local operation of such a process is unique for the density matrices with the rank $n=3$ and 4 , whereas it can be constructed variously for that with $n=2$.

\section{ACKNOWLEDGMENTS}

This work was supported in part by the Postdoctoral Science Foundation and the special funds for Major State Basic Research Project No. G001CB3095 of China.

[1] C.H. Bennett, Phys. Today 48, 24 (1995).

[2] C.H. Bennett, H.J. Bernstein, S. Popescu, and B. Schumacher, Phys. Rev. A 53, 2046 (1996).

[3] C. H. Bennett, G. Brassard, S. Popescu, B. Schumacher, J.A. Smolin, and W.K. Wootters, Phys. Rev. Lett. 76, 722 (1996).

[4] M.A. Nielsen, Phys. Rev. Lett. 83, 436 (1999).

[5] G. Vidal, Phys. Rev. Lett. 83, 1046 (1999).

[6] D. Jonathan and M.B. Plenio, Phys. Rev. Lett. 83, 1455 (1999).

[7] G. Vidal, Journ. of Mod. Opt. 47, 355 (2000).

[8] N. Gisin, Phys. Lett. A 210, 151 (1996).

[9] M. Horodecki, P. Horodecki, and R. Horodecki, Phys. Rev. Lett. 78, 574 (1997).

[10] N. Linden, S. Massar, and S. Popescu, Phys. Rev. Lett. 81, 3279 (1998).

[11] A. Kent, N. Linden, and S. Massar, Phys. Rev. Lett. 83 , 2656 (1999).

[12] L.-X. Cen, F.-L. Li, and S.-Y. Zhu, Phys. Lett. A 275, 368 (2000).

[13] F. Verstraete, J. Dehaene, and B. DeMoor, Phys. Rev. A 64, 010101 (2001).

[14] R.T. Thew and W.J. Munro, Phys. Rev. A 64, 022320 (2001).

[15] C.H. Bennett, D.P. DiVincenzo, J. Smolin, and W.K. Wootters, Phys. Rev. A 54, 3824 (1996).

[16] R. Horodecki and M. Horodecki, Phys. Rev. A 54, 1838 (1996).

[17] W.K. Wootters, Phys. Rev. Lett. 80, 2245 (1998).

[18] For the states with rank $n=4$, there exists $\left\langle x_{4} \mid \tilde{x}_{4}\right\rangle \neq$ 0 . Since if $\left\langle x_{4} \mid \tilde{x}_{4}\right\rangle=0$, the state $\left|\tilde{x}_{4}\right\rangle$ shall be orthogonal to all the four linearly-independent states $\left|x_{i}\right\rangle$ $(i=1, \cdots, 4)$. This obviously cannot happen in the 4 dimensional Hilbert space. 